\title{
Fault Diagnosis in Circuits and Systems by Nonparametric Methods
}

\author{
Alexey Zhirabok, Alexey Shumsky \\ Far Eastern Federal University \\ Sukhanova 8, Vladivostok, Russia \\ zhirabok@mail.ru; a.e.shumsky@yandex.com
}

\begin{abstract}
The problem of fault diagnosis in engineering systems with uncertainties described by nonlinear discrete-time models is studied within the scope of analytical redundancy concept. Solution of the problem assumes the checking redundancy relations existing among system inputs and outputs measured over a finite time window. The nonparametric method is used to construct redundancy relations involving transformation of the initial system model into canonical form with special properties. The obtained results are illustrated by the general electric servoactuator of manipulation robots.
\end{abstract}

Keywords: Nonlinear Models, Discrete-Time, Fault Detection and Isolation, Nonparametric Method.

\section{Introduction}

This work is devoted to the problem of fault diagnosis (FD) in different engineering systems including dynamic systems and electrical circuits. Electrical circuits are convenient models for describing different electrical devices such as transformers, synchronous and asynchronous electrical machines, drives, and so on. The feature of such systems and circuits is that they contain non-smooth nonlinearities such as Coulomb friction, saturation, and hysteresis. In the past decades several approaches to the diagnosis in systems and circuits have been developed, see for example [1-7] but most of them do not allow to solve the FD problem in system with non-smooth nonlinearities. One objective of this paper is to consider the approach which can be used for diagnosis both in dynamic systems and electrical circuits based on the model in the form of difference equations containing non-smooth nonlinearities.

This approach is developed within the framework of analytical redundancy concept. According to this concept, FD is based on checking relations that exist among system inputs and outputs measured over a finite time window and generating the residual to make a decision about faults. There are two main methods to generate the residual: closed-loop techniques which involve diagnostic observers for residual generation [5] and open loop techniques when the residual generator is specified in the form of input-output redundancy relations [3].

As soon as modeling uncertainty and faults both may act upon the residual, the robustness problem arises. It is common practice to distinguish active and passive approaches to solve this problem. Among the active approaches, there exists the method considered in [8-12]. The feature of this method is that knowledge of some system parameters values is not required for redundancy relations checking. Therefore, this method can be called as the nonparametric one.

The feature of the solution obtained in [8-10] is that to check the redundancy relations, it is necessary to find a kernel of some matrix of functionals which is constructed on-line by processing the system inputs and outputs measured over a finite time window.

The objective of the present work is to extend the nonparametric method given in [8-10] to systems and circuits described by nonlinear discrete-time models containing non-smooth nonlinearities. Besides, a new method to obtain the input-output representation of the system under diagnosis is suggested; such a representation is required to apply the nonparametric method.

\section{Basic Relations}

Consider nonlinear difference model

$$
x(t+1)=f(x(t), u(t), \gamma(t)), \quad y(t)=h(x(t)) .
$$


Here $x \in R^{n}, u \in R^{m}, y \in R^{l}$ are the states, controls and outputs, respectively; $\gamma=\left(\gamma_{1}, \ldots, \gamma_{s}\right)^{\mathrm{T}}$ is the vector of parameters; the $i$-th fault is presented as inadmissible deviation of the $i$-th parameter $\gamma_{i}$ from its nominal value $\gamma_{0 i}$, $i=1,2, \ldots, s ; f$ and $h$ are the nonlinear functions, the function $f$ may be non-smooth.

Clearly, equations (1) describe both wide class of technical systems and electric circuits. For circuits, such equations can be obtained based on Kirchhoff's laws and the equations $C \dot{U}=I$ and $L \dot{I}=U$ describing the voltages across the capacitors and inductors and the appropriate currents. As a result, one obtains differential equations which can be transformed into the difference form (1) by using the well-known Euler discretization.

To solve the FD problem by the nonparametric method, one has to transform the vectors of states and outputs as $x_{*}=\varphi(x)$ and $y_{*}=\psi(y)$ using some functions $\varphi$ and $\psi$ such that the general model in new coordinates

$$
\begin{gathered}
x_{*}(t+1)=f_{*}\left(x_{*}(t), u(t), y(t)\right), \\
y_{*}(t)=h_{*}\left(x_{*}(t)\right)
\end{gathered}
$$

is feedback-free:

$$
\begin{aligned}
& x_{* i}(t+1)=f_{*}{ }^{(i)}\left(x_{* i+1}(t), y(t), u(t)\right), \quad i=1,2, \ldots, k-1, \quad x_{* k}(t+1)=f_{*}{ }^{(k)}(y(t), u(t)), \\
& y_{*}(t)=h_{*}\left(x_{* 1}(t)\right)
\end{aligned}
$$

where $x_{* i}$ is the $i$-th component of the state vector $x_{*} \in R^{k}, f_{*}^{(1)}, \ldots, f_{*}^{(k)}$, and $h_{*}$ are some functions. There exists more general feedback-free form; the model (3) is used for simplicity. Based on (3), one constructs the input-output representation of the model (2) as follows.

Make in (3) several temporal shifts and substitutions:

$$
\begin{aligned}
& x_{* 1}(t+2)=f_{*}^{(1)}\left(x_{* 2}(t+1), y(t+1), u(t+1)\right)=f_{*}^{(1)}\left(f_{*}^{(2)}\left(x_{* 3}(t), y(t), u(t)\right), y(t+1), u(t+1)\right), \\
& x_{* 1}(t+3)=f_{*}^{(1)}\left(f_{*}^{(2)}\left(f_{*}^{(3)}\left(x_{* 4}(t), y(t), u(t)\right), y(t+1), u(t+1)\right), y(t+2), u(t+2)\right), \\
& \cdots \\
& \left.\left.y_{*}(t+k)=h_{*}\left(x_{* 1}(t+k)\right)=F_{*}(y(t), u(t)), y(t+1), u(t+1)\right), \ldots, y(t+k-1), u(t+k-1)\right)
\end{aligned}
$$

for some function $F_{*}$. Assume that this function is of the form

$$
\left.\left.F_{*}=\sum_{i=1}^{p} \Gamma_{i}(\gamma) P_{i}(y(t), u(t)), y(t+1), u(t+1)\right), \ldots, y(t+k-1), u(t+k-1)\right)
$$

for some $p$ where $\Gamma_{i}(\gamma)$ is an algebraic expression dependent on components of the vector of parameters $\gamma$ whose values may be unknown; the function $P_{i}(*)$ may contain the elements of the vector $\gamma$ whose values are known, $i=1,2, \ldots, p$. Notice that such a representation can always be obtained if $F_{*}$ is polynomial function. If the function $P_{i}(*)$ contains the parameter with unknown value, then to apply the non-parametric method, it should be presented by power series with coefficients dependent on these parameters.

It follows from (5) that the last expression in (4) can be written in the form 


$$
y_{*}(t+k)=\left(\begin{array}{lll}
\Gamma_{1}(\gamma) & \cdots & \Gamma_{p}(\gamma)
\end{array}\right)\left(\begin{array}{c}
P_{1}^{*}(t, \ldots, t+k-1) \\
\ldots \\
P_{p}^{*}(t, \ldots, t+k-1)
\end{array}\right)
$$

where $\left.\left.P_{i}^{*}(t, \ldots, t+k-1):=P_{i}(y(t), u(t)), y(t+1), u(t+1)\right), \ldots, y(t+k-1), u(t+k-1)\right), i=1,2, \ldots, p$. Based on the last formula, write down the expression for $y_{*}$ for $T$ instants of time:

$$
\begin{aligned}
& Y_{T}(t)=\left(\begin{array}{lll}
y_{*}(t) & \cdots & y_{*}(t-T+1)
\end{array}\right)=\left(\begin{array}{llll}
\Gamma_{1}(\gamma) & \cdots & \Gamma_{p}(\gamma)
\end{array}\right) P_{T}(t), \\
& P_{T}(t)=\left(\begin{array}{ccc}
P_{1}^{*}(t-k, \ldots, t-1) & \cdots & P_{1}^{*}(t-k-T+1, \ldots, t-T) \\
\ldots & \cdots & \cdots \\
P_{p}^{*}(t-k, \ldots, t-1) & \cdots & P_{p}^{*}(t-k-T+1, \ldots, t-T)
\end{array}\right) .
\end{aligned}
$$

The feature of (7) is that the parameters with unknown values are in the row $\left(\Gamma_{1}(\gamma) \cdots \Gamma_{p}(\gamma)\right)$, the matrix $P_{T}(t)$ depend on the measured control and outputs and elements of the vector $\gamma$ which values are known. This allows implementing the FD process based on the row $Y_{T}(t)$ and the matrix $P_{T}(t)$ without knowledge of values of some system parameters.

The size of time window $T$ in [8-10] is chosen minimal such that $\operatorname{rank}\left(P_{T}(t)\right)=\operatorname{rank}\left(P_{T-1}(t)\right)$. It follows from this equality that the last column of the matrix $P_{T}(t)$ linearly depends on other columns, i.e. the nonzero vector $v(T)$ exists such that $P_{T}(t) v(T)=0$; this means that the matrix $P_{T}(t)$ has nonempty kernel. As a result, (7) yields $Y_{T}(t) v(T)=0$, which can be considered as a parity relation.

It follows from all above that the rule $r_{T}(t)=Y_{T}(t) v(T), v(T) \in \operatorname{ker}\left(P_{T}(t)\right)$ is robust since it is constructed without knowledge of values of some system parameters. Notice that the residual should be generated on-line unlike the methods based on full description of the system when some calculations can be performed in advance. To reduce the calculation complexity, one may take $T$ such that the number of columns of the matrix $P_{T}(t)$ is bigger than the number of its rows, i.e. $T \geq p+1$; here the equality $\operatorname{rank}\left(P_{T}(t)\right)=\operatorname{rank}\left(P_{T-1}(t)\right)$ is valid. In some particular cases, the value of $T$ may be reduced.

\section{Problem Solution}

To solve the problem of fault isolation, one has to construct a bank of expressions in the form (6), therewith each expression is invariant with respect to some set of faults (parameters) and sensitive to others. Solution of this problem will be found in two steps. On the first step, the model is invariant with respect to some set of faults and having the general form (2) is constructed. On the second step, this model is transformed into the expression (6) and then into (7).

\subsection{Model Invariant with Respect to the Fault Design}

To implement the first step, we use so-called logic-dynamic approach which allows to solve the problem by methods of linear algebra [11, 12]. To apply this approach, the initial system (1) should be represented in the form

$$
x(t+1)=F x(t)+G u(t)+C\left(\begin{array}{c}
\varphi_{1}\left(A_{1} x(t), u(t)\right) \\
\ldots \\
\varphi_{q}\left(A_{q} x(t), u(t)\right)
\end{array}\right)+\sum_{i=1}^{s} D_{i} d_{i}(t), \quad y(t)=H x(t)
$$


where $F, G$, and $C$ are constant matrices under nominal values of the parameters; $H, D_{1}, \ldots, D_{s}$ are constant matrices; $d_{1}(t), \ldots, d_{s}(t)$ are scalar functions describing faults: if the $i$-th parameter has nominal value, then $d_{i}(t)=0$, otherwise $d_{i}(t)$ becomes an unknown function of time, $i=1,2, \ldots, s ; \varphi_{1}, \ldots, \varphi_{q}$ are arbitrary nonlinearities, $A_{1}, \ldots, A_{q}$ are row matrices. The model (8) can be obtained from (1) by some state transformations described in [11, 12].

The logic-dynamic approach has three steps $[11,12]$. On the first step, one removes the nonlinear term from (8), then the problem is solved for linear system with additional linear restriction; on the third step, the obtained linear solution is supplemented by the transformed nonlinear term.

Now we describe the solution on the second step where the linear model is presented in the canonical form which is feedback-free given by

$$
\begin{gathered}
x_{* i}(t+1)=x_{* i+1}+J_{i} y(t)+G *_{i} u(t), \quad i=1,2, \ldots, k-1, \\
x_{* k}(t+1)=J_{k} y(t)+G_{* k} u(t), \\
y_{*}(t)=R y(t)=x_{* 1}(t)
\end{gathered}
$$

where $x_{*}(t)=\Phi x(t), y_{*}(t)=R y(t)$ for some matrices $\Phi$ and $R$. It is known that matrices describing the linear part of the model (8) and the model (9) satisfy the following equations [11-13]:

$$
\Phi_{1}=R H, \quad \Phi_{i} F=\Phi_{i+1}+J_{i} H, \quad G_{*}=\Phi G
$$

where $\Phi_{i}$ and $J_{i}$ are the $i$-th row of the matrices $\Phi$ and $J, i=1,2, \ldots, k, k$ is dimension of the model. The additional restriction on the matrix $\Phi$ caused by the nonlinear term is of the form $A=A_{*}\left(\Phi^{\mathrm{T}} H^{\mathrm{T}}\right)^{\mathrm{T}}$ for some matrix $A_{*}$, where $A=\left(\begin{array}{llll}A_{1}^{\mathrm{T}} & \ldots & A_{q}^{\mathrm{T}}\end{array}\right)^{\mathrm{T}}, A_{*}=\left(\begin{array}{lll}A_{* 1}^{\mathrm{T}} & \ldots & A_{*}^{\mathrm{T}} q\end{array}\right)^{\mathrm{T}}$. The last equality is equivalent to the condition

$$
\operatorname{rank}\left(\Phi^{\mathrm{T}} \quad H^{\mathrm{T}}\right)=\operatorname{rank}\left(\Phi^{\mathrm{T}} \quad H^{\mathrm{T}} \quad A^{\mathrm{T}}\right)
$$

To construct the model independent of $\gamma_{i}$, one has to take into account the condition $\Phi D_{i}=0$. This condition yields the equation $[11,12]$

$$
\begin{aligned}
& \left(\begin{array}{llll}
R & -J_{1} & \cdots & -J_{k}
\end{array}\right)\left(V^{(k)} \quad B^{(k)}\right)=0, \\
& V^{(k)}=\left(\begin{array}{c}
H F^{k} \\
H F^{k-1} \\
\cdots \\
H
\end{array}\right), \quad B^{(k)}=\left(\begin{array}{ccccc}
H D & H F D & H F^{2} D & \cdots & H F^{k-1} D \\
0 & H D & H F D & \cdots & H F^{k-2} D \\
\cdots & \cdots & \cdots & \cdots & \cdots \\
0 & 0 & 0 & \cdots & 0
\end{array}\right) .
\end{aligned}
$$

Equation (12) is solvable with minimal $k$ satisfying the condition $\operatorname{rank}\left(V^{(k)} \quad B^{(k)}\right)<l(k+1)$. If it is valid for some

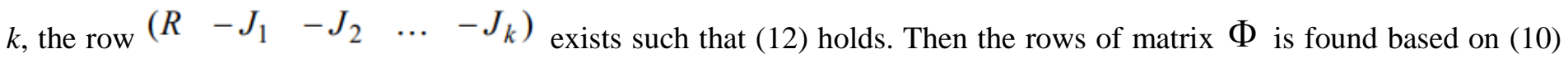
and the condition (11) is checked. If it is satisfied, the matrix $G_{*}=\Phi G$ is calculated and the model (9) has been constructed. If (11) is not satisfied, another solution of (12) is found with former or increased dimension $k$. If (12) is not satisfied for all $k<n$, then the model (9) independent of $\gamma_{i}$ does not exist. In that follows we assume that the condition (11) is satisfied. 
To construct the nonlinear term, notice that it is described by the expression

$C_{*}\left(\begin{array}{c}\varphi_{1}\left(A_{*_{1}} z(t), u(t)\right) \\ \cdots \\ \varphi_{q}\left(A_{*_{q}} z(t), u(t)\right)\end{array}\right)$

where $z=\left(x_{*}^{\mathrm{T}} y^{\mathrm{T}}\right)^{\mathrm{T}}$, the row $A_{*_{i}}$ is found from the equation $A_{i}=A_{*_{i}}\left(\Phi^{\mathrm{T}} H^{\mathrm{T}}\right)^{\mathrm{T}}, i=1,2, \ldots, q, C_{*}=\Phi C$. As a result, the model invariant with respect to $\gamma_{i}$ is of the general form

$$
\begin{aligned}
& x_{* i}(t+1)=f_{*_{i}}\left(x_{*}(t), y(t), u(t)\right), \quad i=1,2, \ldots, k-1, \quad x_{* k}(t+1)=f_{* k}\left(x_{*}(t), y(t), u(t)\right), \\
& y_{*}(t)=x_{* 1}(t)
\end{aligned}
$$

for some functions $f_{* 1}, \ldots, f_{* k}$.

\subsection{Input-output Expression Design}

To obtain the expression of the form (6), for simplicity we consider case $k=3$ in (13) and use the following notations: $x_{*}^{+}=x_{* 1}(t+1), x_{*}=x_{*}(t), u=u(t), y=y(t)$ :

$$
\begin{aligned}
& x_{* 1}^{+}=f_{* 1}\left(x_{*}, y, u\right)=f_{*_{1}}\left(y_{*}, x_{* 2}, x_{* 3}, y, u\right), \quad x_{* 2}^{+}=f_{* 2}\left(x_{*}, y, u\right)=f_{* 2}\left(y_{*}, x_{* 2}, x_{* 3}, y, u\right), \\
& x_{* 3}^{+}=f_{* 3}\left(x_{*}, y, u\right)=f_{* 3}\left(y_{*}, x_{* 2}, x_{* 3}, y, u\right), \quad y_{*}=x_{* 1} .
\end{aligned}
$$

Make two temporal shifts in the last expression:

$$
y_{*}^{+}=x_{* 1}^{+}=f_{*_{1}}\left(y_{*}, x_{* 2}, x_{* 3}, y, u\right), \quad y_{*}^{++}=f_{* 1}\left(y_{*}^{+}, f_{*_{2}}\left(y_{*}, x_{*_{2}}, x_{* 3}, y, u\right), f_{*_{3}}\left(y_{*}, x_{* 2}, x_{* 3}, y, u\right), y^{+}, u^{+}\right) .
$$

Assume that $\operatorname{rank}\left(\partial \hat{h}_{*}\left(\bar{x}_{*}\right) / \partial \bar{x}_{*}\right)=2$ generically, i.e. everywhere except on the set of zero measure where

$$
\hat{h}_{*}\left(\bar{x}_{*}\right)=\left(\begin{array}{c}
f_{* 1}\left(y_{*}, \bar{x}_{*}, y, u\right) \\
f_{* 1}\left(y_{*}^{+}, f_{* 2}\left(y_{*}, \bar{x}_{*}, y, u\right), f_{* 3}\left(y_{*}, \bar{x}_{*}, y, u\right), y^{+}, u^{+}\right.
\end{array}\right), \quad \bar{x}_{*}=\left(\begin{array}{c}
x_{* 2} \\
x_{* 3}
\end{array}\right) ;
$$

it is assumed that the function $\hat{h}_{*}$ is differentiable with respect to $\bar{x}_{*}$. Under the condition $\operatorname{rank}\left(\partial \hat{h}_{*}\left(\bar{x}_{*}\right) / \partial \bar{x}_{*}\right)=2$ the set of equations (14) is generically solvable for $x_{* 2}$ and $x_{* 3}$ in the form $x_{* 2}=\bar{f}_{* 2}\left(y_{*}, y_{*}^{+}, y_{*}^{++}, y, y^{+}, u, u^{+}\right)$, $x_{* 3}=\bar{f}_{* 3}\left(y_{*}, y_{*}^{+}, y_{*}^{++}, y, y^{+}, u, u^{+}\right)$for some functions $\bar{f}_{* 2}$ and $\bar{f}_{* 3}$.

Make additional temporal shift in $y_{*}^{++}$and then replace the variables $x_{* 2}$ and $x_{* 3}$ in the expression for $y_{*}^{+++}$by the functions $\bar{f}_{* 2}$ and $\bar{f}_{* 3}$, respectively. As a result, the expression in the input-output form has been obtained:

$$
y_{*}^{+++}=R y(t+3)=f_{* *}(R y(t), R y(t+1), R y(t+2), y(t), y(t+1), u(t), u(t+1))
$$

where $f_{* *}$ is some function. 


\subsection{Heuristic Approach}

When the function $\hat{h}_{*}$ is non-differentiable with respect to $\bar{x}_{*}$, one may use some heuristics to transform the model (13) into the form (15). Let (13) with $k=3$ be described by the equations

$$
\begin{aligned}
& x_{* 1}^{+}=x_{* 2}+f_{* 1}\left(x_{* 1}\right)+J_{* 1} y+G_{* 1} u, \quad x_{* 2}^{+}=x_{* 3}+f_{* 2}\left(x_{* 2}\right)+J_{* 2} y+G_{* 2} u, \\
& x_{* 3}^{+}=f_{* 3}\left(x_{* 3}\right)+J_{* 3} y+G_{* 3} u, \quad y_{*}=x_{* 1},
\end{aligned}
$$

where $f_{*_{1}}, f_{*_{2}}, f_{* 3}$ are arbitrary nonlinear functions. The peculiarity of the model is that the right-hand side of the equation for every variable includes nonlinear function of the same variable only.

Make two temporal shifts and substitutions for $y_{*}$ :

$$
\begin{aligned}
& y_{*}^{++}=x_{*_{2}}^{+}+f_{*_{1}}\left(x_{*_{1}}^{+}\right)+J_{*_{1}} y^{+}+G_{*_{1}} u^{+}=f_{*_{1}}\left(y_{*}^{+}\right)+J_{*_{1}} y^{+}+G_{*_{1}} u^{+}+x_{*_{3}}+f_{*_{2}}\left(x_{*_{2}}\right)+J_{*_{2}} y+G_{*_{2}} u, \\
& y_{*}^{+++}=f_{*_{1}}\left(y_{*}^{++}\right)+J_{*_{1}} y^{++}+G_{*_{1}} u^{++}+x_{*_{3}}^{+}+f_{*_{2}}\left(x_{*_{2}}^{+}\right)+J_{*_{2}} y^{+}+G_{*_{2}} u^{+}= \\
& f_{*_{1}}\left(y_{*}^{++}\right)+J_{*_{1}} y^{++}+J_{*_{2}} y^{+}+G_{*_{1}} u^{++}+G_{*_{2}} u^{+}+f_{*_{2}}\left(x_{*_{2}}^{+}\right)+f_{*_{3}}\left(x_{*_{3}}\right)+J_{*_{3}} y+G_{*_{3}} u .
\end{aligned}
$$

Find the sum $x_{* 3}+f_{*_{2}}\left(x_{*_{2}}\right)+J_{*_{2}} y+G_{*_{2}} u=: x_{*_{2}}^{+}$from the formula for $y_{*}^{++}: x_{* 2}^{+}=y_{*}^{++}-f_{* 1}\left(y_{*}^{+}\right)-J_{* 1} y^{+}-G_{* 1} u^{+}$ and use it as an argument of the function $f_{* 2}$ in the equation for $y_{*}^{+++}$:

$$
\begin{aligned}
& y_{*}^{+++}=f_{*_{1}}\left(y_{*}^{++}\right)+J_{*_{1}} y^{++}+J_{*_{2}} y^{+}+G_{* 1} u^{++}+G_{* 2} u^{+}+ \\
& +f_{*_{2}}\left(y_{*}^{++}-f_{*_{1}}\left(y_{*}^{+}\right)-J_{*_{1}} y^{+}-G_{*_{1}} u^{+}\right)+f_{*_{3}}\left(x_{* 3}\right)+J_{* 3} y+G_{*_{3}} u .
\end{aligned}
$$

Next, find the sum $f_{* 3}\left(x_{* 3}\right)+J_{* 3} y+G_{* 3} u$ from the last expression, construct the expression for $y_{*}^{++++}$and use this sum as an argument of the function $f_{* 3}$, then the final expression for $y_{*}^{++++}$contains the temporal shifts of the vectors $y_{*}=R y, y$ and $u$, that is necessary for the non-parametric method.

\section{Practical Example}

Consider the discretized model of the general electric servoactuator of manipulation robots under absence of the external loading moment:

$$
\begin{aligned}
& x_{1}^{+}=\gamma_{1} x_{2}+x_{1}, \\
& x_{2}^{+}=\gamma_{2} x_{3}+\gamma_{3} \operatorname{sign}\left(x_{2}\right), \\
& x_{3}^{+}=\gamma_{4} x_{2}+\gamma_{5} x_{3}+\gamma_{6} u,
\end{aligned}
$$

where $x_{1}$ is the output rotation angle at the reducer output shaft; $x_{2}$ is the output rotation velocity at the motor output shaft; $x_{3}$ is the current through the servoactuator windings; the coefficients $\gamma_{1} \div \gamma_{6}$ are given for the general electric servoactuator parameters and the sampling time. We assume that $y_{1}=x_{1}$ and $y_{2}=x_{3}$.

It follows from (16) that the coefficients $\gamma_{2}, \gamma_{3}$ and $\gamma_{4} \div \gamma_{6}$ have the same influence on $x_{2}^{+}$and $x_{3}^{+}$, respectively, therefore one has three sets of indistinguishable faults: $K_{2}=\left\{\gamma_{1}\right\}, K_{2}=\left\{\gamma_{2}, \gamma_{3}\right\}$, and $K_{3}=\left\{\gamma_{4}, \gamma_{5}, \gamma_{6}\right\}$.

The logic-dynamic description of the model (16) is as follows: 
$F=\left(\begin{array}{ccc}1 & \gamma_{1} & 0 \\ 0 & 1 & \gamma_{2} \\ 0 & \gamma_{4} & \gamma_{5}\end{array}\right), \quad G=\left(\begin{array}{c}0 \\ 0 \\ \gamma_{6}\end{array}\right), \quad H=\left(\begin{array}{ccc}1 & 0 & 0 \\ 0 & 0 & 1\end{array}\right), \quad C=\left(\begin{array}{c}0 \\ \gamma_{3} \\ 0\end{array}\right), \quad D_{1}=\left(\begin{array}{l}1 \\ 0 \\ 0\end{array}\right), \quad D_{2}=\left(\begin{array}{l}0 \\ 1 \\ 0\end{array}\right), \quad D_{3}=\left(\begin{array}{l}0 \\ 0 \\ 1\end{array}\right)$, $A=\left(\begin{array}{lll}0 & 1 & 0\end{array}\right)$.

Remove the nonlinear term from the model (16) and consider its linear part for the first fault. It can be shown that (12) has a solution $\left(\begin{array}{lll}R-J_{1} & -J_{2}\end{array}\right)=\left(\begin{array}{llllll}0 & 1 & 0 & -\left(1+\gamma_{4}\right) & 0 & -\left(\gamma_{2} \gamma_{4}-\gamma_{5}\right.\end{array}\right)$, then $\quad \Phi_{1}=\left(\begin{array}{lll}0 & 0 & 1\end{array}\right)$, $\Phi_{2}=\left(\begin{array}{lll}0 & \gamma_{4} & -1\end{array}\right), G_{*}=\left(\begin{array}{ll}\gamma_{6} & -\gamma_{6}\end{array}\right)^{\mathrm{T}}$. It can be checked that the condition (11) is satisfied and the matrix $A_{*}$ is of the form $A_{*}=\left(\begin{array}{llll}0 & 1 / \gamma_{4} & 0 & 1 / \gamma_{4}\end{array}\right)^{\mathrm{T}}$; besides $C_{*}=\Phi C=\left(\begin{array}{ll}0 & \gamma_{3} \gamma_{4}\end{array}\right)^{\mathrm{T}}$. As a result, one obtains the model

$$
\begin{aligned}
& x_{* 1}^{+}=x_{* 2}+\left(1+\gamma_{5}\right) y_{2}+\gamma_{6} u, \\
& x_{* 2}^{+}=\left(\gamma_{2} \gamma_{4}-\gamma_{5}\right) y_{2}-\gamma_{6} u+\gamma_{3} \gamma_{4} \operatorname{sign}\left(\left(y_{2}+x_{* 2}\right) / \gamma_{4}\right), \\
& y_{*}=x_{* 1}=y_{2},
\end{aligned}
$$

where $x_{* 1}:=x_{3}, x_{* 2}:=\gamma_{2} x_{2}-x_{3}$. To obtain the feedback-free expression, make two temporal shifts:

$$
\begin{aligned}
& y_{2}^{+}=x_{* 2}+\left(1+\gamma_{5}\right) y_{2}+\gamma_{6} u, \\
& y_{2}^{++}=\left(\gamma_{2} \gamma_{4}-\gamma_{5}\right) y_{2}-\gamma_{6} u+\gamma_{3} \gamma_{4} \operatorname{sign}\left(\left(y_{2}+x_{* 2}\right) / \gamma_{4}\right)+\left(1+\gamma_{5}\right) y_{2}^{+}+\gamma_{6} u^{+} .
\end{aligned}
$$

It follows from the expression for $y_{2}^{++}$that the sum of three first addends may be presented as $y_{2}^{++}-\left(1+\gamma_{5}\right) y_{2}^{+}-\gamma_{6} u^{+}$. Taking this into account, write down the expression for $y_{2}^{+++}$:

$$
y_{2}^{+++}=\left(\gamma_{2} \gamma_{4}-\gamma_{5}\right) y_{2}^{+}-\gamma_{6} u^{+}+\left(1+\gamma_{5}\right) y_{2}^{++}+\gamma_{6} u^{++}+\gamma_{3} \gamma_{4} \operatorname{sign}\left(\left(y_{2}^{+}+y_{2}^{++}-\left(1+\gamma_{5}\right) y_{2}^{+}-\gamma_{6} u^{+}\right) / \gamma_{4}\right)
$$

Since the function sign contains the parameters $\gamma_{4} \div \gamma_{6}$, then to implement the nonparametric method, their values should be known.

The expressions for other shifts can be obtained by analogy. Since (17) contains 5 addends, $p=5$ and $T=6$. Denote the function $\operatorname{sign}(*)$ in (17) via $w(t)$ and write down it in the form (7) with $y_{*}=R y=y_{2}$ :

$$
\begin{aligned}
& Y_{6}(t)=\left(\begin{array}{llllll}
y_{2}(t) & y_{2}(t-1) & y_{2}(t-2) & y_{2}(t-3) & y_{2}(t-4) & y_{2}(t-5)
\end{array}\right)=
\end{aligned}
$$

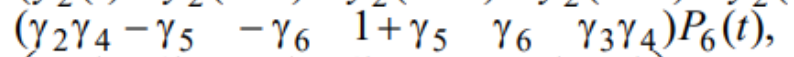

$$
\begin{aligned}
& P_{6}(t)=\left(\begin{array}{cccc}
y_{2}(t-2) & y_{2}(t-3) & \cdots & y_{2}(t-7) \\
u(t-2) & u(t-3) & \cdots & u(t-7) \\
y_{2}(t-1) & y_{2}(t-2) & \cdots & y_{2}(t-6) \\
u(t-1) & u(t-2) & \cdots & u(t-6) \\
w(t-3) & w(t-4) & \cdots & w(t-8)
\end{array}\right) .
\end{aligned}
$$

The residual is generated as $r_{6}(t)=Y_{6}(t) v(T), v(6) \in \operatorname{ker}\left(P_{6}(t)\right)$. By analogy, other faults are considered and a bank of expressions similar to (18) is constructed to isolate faults. 


\section{Conclusion}

The paper considers the problem of fault diagnosis in dynamic systems and electrical circuits described by nonlinear models with non-smooth nonlinearities. So-called nonparametric method to solve this problem has been used. The feature of this method is that some parameters of the system under consideration may be unknown. Theoretical results are illustrated by practical example.

\section{Acknowledgements}

This paper was supported by the Russian Scientific Foundation (project 16-19-00046).

\section{References}

[1] F. Aminian, M. Aminian, and H. Collins, "Analog fault diagnosis of actual circuits using neural networks," IEEE Trans. Instrumentation and Measurement, vol. 51, pp. 544-550, 2002.

[2] J. Benlder and A. Salama, "Fault diagnosis in analog circuits," Proc. IEEE, vol. 73, pp. 1279-1325, 1985.

[3] M. Blanke, M. Kinnaert, J. Lunze, and M. Staroswiecki, Diagnosis and fault-tolerant control. Berlin: Springer-Verlag, 2006.

[4] L. Chen and R. Patton, Robust model-based fault diagnosis for dynamic systems. Norwell: Kluwer Academic Publishers, 1999.

[5] S. Ding, Model-based fault diagnosis techniques - design schemes, algorithms and tools. London: Springer-Verlag, 2013.

[6] V. Prasannamoorthy and N. Devarajan, "Fault detection and classification in power electronic circuits using wavelet transform and neural network," Journal of Computer Science, vol. 7, pp. 95-100, 2011.

[7] A. Zhirabok, V. Filaretov and D. Tkachev, "Non-parametric method for fault diagnosis in electrical circuits," in Proceedings of 23d Int. DAAAM Symposium, Croatia, pp. 5-8, 2012.

[8] S. Ding, Data-driven design of fault diagnosis and fault-tolerant control systems. London: Springer-Verlag, 2014.

[9] A. Haghani, M. Krueger, T. Jeinsch, S. Ding, and P. Engel, "Data-driven multimode fault detection for wind energy conversion systems," in Proceedings of 9th IFAC Symposium Safeprocess'2015, France, 2015, pp. 633-638.

[10] A. Shumsky, "Redundancy relations for fault diagnosis in nonlinear uncertain systems," Int. J. Applied Mathematics and Computer Science, vol. 17, pp. 477-489, 2007.

[11] A. Zhirabok and A. Shumsky, "Fault diagnosis in nonlinear dynamic systems by non-parametric method," in Proceedings of 25th Mediterranean Conf. on Control and Automation, Malta, pp. 424-429, 2017.

[12] A. Zhirabok and A. Shumsky, "Non-parametric method for fault isolation in nonlinear dynamic systems," in Proceedings of 20th World IFAC Congress, France, pp. 2990-2995, 2017.

[13] P. Frank, "Fault diagnosis in dynamic systems using analytical and knowledge-based redundancy. A survey and some new results," Automatica, vol. 26, pp. 459-474, 1990. 